\title{
Corneal Sensory Experience via Transient Receptor Potential Vanilloid 1 Accelerates the Maturation of Neonatal Tearing
}

\author{
Kai Jin, ${ }^{*}$ Toshihiro Imada, ${ }^{*}$ Shigeru Nakamura, ${ }^{*}$ Yusuke Izuta, ${ }^{*}$ Erina Oonishi, ${ }^{*}$ Michiko Shibuya, ${ }^{*}$ Hisayo Sakaguchi, \\ Hirotaka Tanabe, ${ }^{*}$ Masataka Ito, ${ }^{\dagger}$ Kimiaki Katanosaka, ${ }^{\ddagger}$ and Kazuo Tsubota*
}

\begin{abstract}
From the Department of Ophthalmology, * Keio University School of Medicine, Tokyo; the Department of Developmental Anatomy and Regenerative Biology, ${ }^{\dagger}$ National Defense Medical College, Tokorozawa; and the Department of Biomedical Sciences, ${ }^{\ddagger}$ College of Life and Health Sciences, Chubu University, Kasugai, Japan
\end{abstract}

\author{
Accepted for publication \\ May 22, 2019. \\ Address correspondence to \\ Shigeru Nakamura D.V.M., \\ Ph.D., or Kazuo Tsubota, M.D., \\ Department of Ophthalmology, \\ Keio University School of \\ Medicine, 35 Shinanomachi, \\ Shinjuku, Tokyo 160-8582, Jap- \\ an. E-mail: s-nakamura.a5@ \\ keio.jp or tsubota@z3.keio.jp.
}

\begin{abstract}
Tearing maturates rapidly after birth, and external environmental challenges play a key role in promoting lacrimal functional maturation. However, little is known about the facilitative factors underlying this developmental process or the potential of application of these factors to treat hypofunction of the lacrimal gland. In this study, eye opening and the subsequent ocular surface sensory experience, which is thought to be involved in postnatal maturation of lacrimal function, were investigated. Our results demonstrated that eye opening after birth is essential for the maturation of neonatal tearing. The maturation process of lacrimal function is dependent on the ocular surface sensory experience via transient receptor potential cation channel subfamily member 1 after birth. This study provides, for the first time, important evidence of the sensory experience of the ocular surface in relation to the maturation of functional tear secretion during the postnatal period. (Am J Pathol 2019, 189: 1699-1710; https://doi.org/10.1016/j.ajpath.2019.05.015)
\end{abstract}

When an infant is born, the first challenges that must be faced and completed compose a critical period of physiological adaptations. During this time, the gradual and progressive maturation of effective ventilation, closed circulation, energy absorption, and the nervous system allows the newborn to deal with the sudden drastic changes in the environment and to move from reliance on the maternal resource. ${ }^{1-3}$ These vulnerable newborns cry for care, support, and protection, resulting from an interaction of activity of certain brain structures, neurotransmitters, and hormones, to help them go through the adaptation time. ${ }^{4}$

Compared with the relatively complex biopsychosocial concept of crying or weeping, ${ }^{4}$ the physiological aspect of tearing mainly refers to the process where the fluid mixture produced by the lacrimal gland (LG) is secreted and expelled onto the ocular surface to lubricate, nourish, and protect the ocular surface, as a result of the multifunctional characteristics of the tear film, as well as to maintain a uniform and regular refractive surface for optimal optical quality. $^{5-7}$ Normal infants cry without tearing during the first 2 weeks after birth, and the tear secretion of newborns is lower than that of infants or adults, a phenomenon that can be traced back to Aristotle (Historia Naturalis). ${ }^{8,9}$ The current understanding of neonatal tearing during the postnatal period based on observational and clinical evaluation approaches showed that the basal tear volume of newborns without any stimulation is limited but increases rapidly in early infancy. ${ }^{10}$ Preterm infants have reduced reflex and basal tear secretion. ${ }^{11}$ Accumulating evidence indicates that the vital organs are still immature when infants are born. However, the mechanisms that underlie the maturation of LG and neonatal tearing, in response to environmental changes during a brief period after birth, remain largely unknown.

Supported by Grants-in-Aid for Scientific Research (KAKENHI) grant 17K16983 (T.I.).

K.J. and T.I. contributed equally to this work.

Disclosures: None declared. 
Sensory experience is information perceived from sensing the environment and changes surrounding an organism and is performed by sensory organs, including the eyes, ears, nose, tongue, and skin. ${ }^{12,13}$ This information is collected from sensory receptors that are located throughout the body, processed, and stored by networks of neurons. ${ }^{12-14}$ The structure of the sensory circuits is formed by individual genetic conditions, as well as the presence and enhancement of neural activity induced by sensory experience, and helps to refine the functional output of the target organs. ${ }^{12-14}$

The cornea is the most densely innervated tissue in the body. ${ }^{15-17}$ Ocular surface sensory experiences are evoked by distinct functional types of corneal sensory neurons that are broadly classified as polymodal nociceptor neurons, cold thermoreceptor neurons, or selective mechanonociceptor neurons. ${ }^{15-17}$ Cornea sensitivity is necessary to detect and timely respond to the environmental changes or pathologic stresses, interact with the central nerve system, protect against potential damage, and maintain the healthy integrity of the ocular surface.

Herein, we investigated the effect of eye opening after birth on the maturation of neonatal tearing. We determined whether the ocular surface sensory experience after eye opening plays a significant role in the maturation of LGs by modulating the intensity of ocular surface sensory activity.

\section{Materials and Methods}

\section{Animals}

C57BL/6J wild-type (WT), homozygous, TRPV1 knockout (KO) mice (stock number 3770; The Jackson Laboratory, Bar Harbor, ME $)^{18}$ and homozygous TRPM $8 \mathrm{KO}$ mice (stock number 8198; The Jackson Laboratory), aged from 4 to 28 days, were used in this study. All procedures were performed in accordance with the Association of Research and Vision in Ophthalmology statement for the Use of Animals in Ophthalmology and Vision Research and were approved by the Animal Experimentation Ethics Committee of Keio University School of Medicine (Tokyo, Japan; number 11008) and by the Animal Experiment Committee of Chubu University (Kasugai, Japan; number 2910071). All animals were housed under the general conditions [room temperature was $23^{\circ} \mathrm{C} \pm 2{ }^{\circ} \mathrm{C}$, humidity was $60 \% \pm 10 \%$, there was an alternating 12-hour light-dark cycle (8 Am to 8 PM), and water and food were available ad libitum].

\section{Tarsorrhaphy (Eyelid Suture)}

Eyelid suture was performed binocularly in 10-day-old WT mice under inhalation anesthesia with isoflurane (Pfizer, Tokyo, Japan) by the same operator (K.J.). This procedure involved suturing the unopened eyelids with three interrupted 8-0 stitches (Vicryl; Ethicon Inc., Somerville, MA). The eyelids of the mice were kept closed by sutures until postnatal day (P) 21, after which the eyelid sutures were removed.

\section{Tear Secretion Measurement}

Tear secretion was measured using a modified Schirmer test $^{19,20}$ with a phenol red thread (Zone-Quick; Showa Yakuhin Kako, Tokyo, Japan). This was placed on the temporal side of the conjunctiva between the limbus and the outer canthus for 15 seconds. The length of the moistened area from the edge was measured to within $0.5 \mathrm{~mm}$. To determine the change in tear secretion during the eyelid-suture period, tear secretion was measured just after removing the eyelid suture at P14 and P21 in a separate group.

\section{Two-Photon Visualization of Acinar Cell Surface and Myoepithelial Cells of LGs}

C57BL/6J WT mice at P12 and P21 and eyelid-sutured mice at P21 were euthanized with an overdose of pentobarbital sodium; then, the LGs were dissected.

The LGs were fixed in $4 \%$ paraformaldehyde at $4{ }^{\circ} \mathrm{C}$ overnight. After fixation, LGs were blocked with $1 \%$ bovine serum album in phosphate-buffered saline (PBS) containing $0.25 \%$ Triton X-100 for 1 hour at room temperature and then incubated overnight at $4{ }^{\circ} \mathrm{C}$ with a rabbit polyclonal antibody against calponin, a specific myoepithelial cell marker $^{21,22}$ (1:300 dilution; Abcam, Cambridge, MA). After being washed with PBS, LGs were incubated with Alexa Fluor 488-conjugated donkey-anti-rabbit secondary antibody (1:300 dilution; Molecular Probes, Eugene, OR) for 3 hours at room temperature $\left(25^{\circ} \mathrm{C} \pm 5^{\circ} \mathrm{C}\right)$ and washed with PBS. For visualization of the cell surface, LGs were stained with phalloidin-labeled wheat germ agglutinin (1:500 dilution; Vector Laboratories, Burlingame, CA), which binds to lectins and acts as a marker for the cell surface, ${ }^{23}$ at room temperature for 30 minutes. Wheat germ agglutinin-stained LGs were washed three times with PBS for 3 minutes.

The LG was observed with a two-photon microscope (FV1200MPE; Olympus, Tokyo, Japan) equipped with a water-immersion objective lens $(\mathrm{XLPlaN} 25 \times 1.05 \mathrm{WMP}$; Olympus). The excitation wavelength for wheat germ agglutinin and Alexa-488 was $830 \mathrm{~nm}$, and the emission was simultaneously detected through a bandpass filter for wheat germ agglutinin (575 to $630 \mathrm{~nm}$ ) and for Alexa-488 $(510$ to $550 \mathrm{~nm})$. The fluorescence images at a depth of approximately $200 \mu \mathrm{m}$ from the surface of the LG were reconstructed from images ( $200 \mu \mathrm{m}$ thick) acquired at z-step sizes of $1 \mu \mathrm{m}$ using Imaris 8.4.0 software (Bitplane $A G$, Zurich, Switzerland).

\section{Histopathologic Morphometric Analysis}

For hematoxylin and eosin staining, mice of different postnatal ages (P12 and P21) and eyelid-sutured mice at P21 were euthanized with an overdose of pentobarbital sodium; then, their LGs were dissected. The whole LG was fixed in a $10 \%$ formalin solution and embedded in paraffin. Sections (5 $\mu \mathrm{m}$ thick) were obtained from the middle of the 
horizontal direction in the whole LG. The sections were stained with filtered hematoxylin and eosin. Images were captured using optical microscope BIOREVO BZ-9000 (Keyence, Osaka, Japan). For quantification of the sizes of the acinar cells, the sizes of 20 acinar cells were measured in three randomly selected areas $\left(10,000 \mu \mathrm{m}^{2}\right)$ from each section using BZ-Analyzer 2.1 software (Keyence). For the determination of the acinar cell density, the area of the whole specimens was quantified using BZ-Analyzer software in Hybrid Cell Count mode (Keyence), and the occupied area ratio of the individual acinar cells/whole specimens was calculated in each section.

For transmission electron microscopy, mice at P12 and P21 and eyelid-sutured mice at P21 were perfused with Karnovsky's fixative $(2.5 \%$ glutaraldehyde and $2 \%$ paraformaldehyde in $0.1 \mathrm{~mol} / \mathrm{L}$ sodium cacodylate; $\mathrm{pH}$ 7.4) under anesthesia, and the excised LG was immersed in the fixative. Sections ( $1 \mu \mathrm{m}$ thick) were stained with methylene blue, and ultrathin sections were made using a diamond knife. The ultrathin sections were collected on mesh grids, stained with uranyl acetate and lead citrate, and examined using an electron microscope (JEM-1400Plus; JEOL Ltd, Tokyo, Japan).

\section{RNA Isolation and Quantitative Real-Time PCR}

Total RNA was isolated from the LG using an RNA extraction reagent (ISOGEN; Nippon Gene, Tokyo, Japan), according to the manufacturer's instructions. The RNA was used for reverse transcription; and then, cDNA synthesis was performed using the ReverTra Ace qPCR RT kit (TOYOBO, Osaka, Japan). SYBR Green-based quantitative real-time PCR was performed using the Step One Plus system (Applied Biosystems, Framingham, MA). Each PCR amplification was performed using a specific primer set. The primer sequences were as follows: GAPDH, 5'-ACCCAGAAGACTGTGGATGG-3' (sense) and 5'-GGATGCAGGGATGATGTTCT-3' (antisense); and lactoperoxidase (Lpo), 5'-AAGGCACACTGCAGTGATGAAA- $3^{\prime}$ (sense) and $5^{\prime}$-TTGGCATGCTTGAGATAATCTGAC- $3^{\prime}$ (antisense). Data were normalized to GAPDH.

\section{Tear Protein Analysis}

The tear component proteins extracted from a phenol red thread were diluted in PBS to $0.4 \mathrm{mg} / \mathrm{mL}$ protein concentrations. The same volume of $2 \times$ Laemmli sample buffer was added to the tear samples, as well as $5 \% \beta$-mercaptoethanol. The tear samples were then boiled at $100^{\circ} \mathrm{C}$ and separated by PAGE. After electrophoresis, the gels were stained in 50\% methanol $/ 5 \%$ acetic acid $/ 0.25 \%$ Coomassie brilliant blue R-250 and destained in 5\% methanol/7\% acetic acid. Band intensities were quantified using ImageJ software version 1.52a (NIH, Bethesda, MD; http://imagej. nih.gov/ij).

\section{Stimulation of Corneal Sensory Inputs}

After P14 (when eye opening occurs), mouse pups were treated with eye drops of capsaicin $(1 \mu \mathrm{mol} / \mathrm{L}$; Wako Pure Chemical, Osaka, Japan), menthol (50 $\mu \mathrm{mol} / \mathrm{L}$; Tokyo Chemical, Tokyo, Japan), or vehicle saline solution three times per day for 7 consecutive days. A $1 \mu \mathrm{L}$ drop of capsaicin $(1 \mu \mathrm{mol} / \mathrm{L})$, menthol $(50 \mu \mathrm{mol} / \mathrm{L})$, or vehicle saline solution was softly applied to the center of the cornea using a micropipette.

\section{Suppression of Neonatal Corneal Sensory Inputs}

On P5 and P6, the mouse pups were given a single s.c. injection of capsaicin per day, at a dose of $50 \mathrm{mg} / \mathrm{kg}$, dissolved in saline containing 10\% Tween-80 and 10\% ethanol. Vehicle-treated mouse pups received an injection of saline containing 10\% Tween- 80 and $10 \%$ ethanol at the same dose. The mouse pups injected with capsaicin or saline solution were then housed with their mothers and fed under normal conditions. A sustained decrease of corneal sensitivity in capsaicin-treated neonatal mice was confirmed, as reported in previous studies. ${ }^{24-26}$

\section{Measurement of Wipe Behavior}

Capsaicin $(1 \mu \mathrm{mol} / \mathrm{L})$, menthol $(50 \mu \mathrm{mol} / \mathrm{L})$, or vehicle (saline) was pipetted onto the corneal surface of the mice at the volume of $1 \mu \mathrm{L}$. Immediately after the ocular application of capsaicin or vehicle, the mice were placed into a plexiglass chamber; and the number of ipsilateral eye wipes with the forelimb was counted for 3 minutes. Facial grooming with both forepaws and hind paw scratches were also included. After the assessment of wipe behavior, the mice were removed from the chamber and returned to the initial cage.

\section{Corneal Sensitivity}

Corneal sensitivity was measured without topical anesthesia using a modified Cochet-Bonnet filament aesthesiometer. The testing was initiated using a nylon filament of $4.0 \mathrm{~cm}$. This was the end of the nylon filament used to touch the central part of the cornea. If the mouse blinked, the length of the filament was recorded. If the mouse did not blink, the nylon filament was shortened by $0.5 \mathrm{~cm}$ and the test was repeated until the blinking reflex was observed and recorded. This process was repeated for each eye three times.

To evaluate the changes in corneal sensitivity after repetitive corneal stimulation with eye drops of capsaicin or menthol, measurements were performed before first instillation (P14) and 24 hours after final instillation of capsaicin or menthol (P21).

\section{Statistical Analysis}

Statistical analyses were performed using JMP12 software version 12.2 (SAS Institute, Cary, NC). Comparisons 
between the two groups were performed using an F-test, followed by the $t$-test for parametric variables and the $U$-test for nonparametric variables. Multiple comparisons were performed using a one-way analysis of variance, followed by the Tukey-Kramer or Dunnett test. The differences between the measurement variables were considered significant if $P \leq$ 0.05 .

\section{Results}

\section{Effect of Eye Opening on Lacrimal Gland and Neonatal Tearing}

The time of natural eye opening in the mice was from P12 to P14. Herein, $>95 \%$ of the mouse pups opened their eyes naturally at P14 (Figure 1A), as reported previously. ${ }^{27-29}$ To determine whether the rapid maturation/growth of neonatal tearing that follows eye opening could be attributed solely to endogenous developmental mechanisms, binocular eyelid suture was performed on the mouse pups before the time of eye opening (P10) through $\mathrm{P} 21$, at which point the eyelid suture was removed (Figure 1B). Linear elevation of the body weight was observed in the normal and eyelidsutured group from P12 to P21 at the same level (Figure 1C). In the normal group, tear secretion was gradually increased after eye opening (Figure 1D). A significant increase in tear secretion was observed at P18, P21, and P28 compared with that at P14. In the eyelid-sutured group, tear secretion in the pups that delayed eye opening by 1 week was unchanged at P21 compared with that at P12. At P21, tear secretion was significantly lower in the eyelid-sutured group than in the normal group. Tear secretion in the eyelid-sutured group was increased from P21 to P28, and significance was observed between P21 and P28. No difference in tear secretion was observed at P 28 between the normal and eyelid-sutured groups.

The LG of mouse pups at P7 was small, with a size of approximately $3.0 \mathrm{~mm}$ (vertical) and $2.5 \mathrm{~mm}$ (horizontal). No obvious changes in the LG size were observed before the eye-opening period (P7 to P14) in both the normal and eyelid-sutured groups. During the postnatal age before eye opening, no significant changes in the LG weight were observed (P7 to P14). In the normal mouse pup, the LG weight increased significantly after eye opening and the weight at P21 was approximately fourfold of the weight at P14 (Figure 1E). In the eyelid-sutured mouse pups, the LG weight significantly increased with postnatal age; however, the weight at P21 was significantly lower than that in the age-matched normal mouse pups.

To further assess the relevance of eye opening to $\mathrm{LG}$ maturation, the changing ratio of the weight of LG, other organs, and the whole body was calculated at P21 versus those at P12. In the normal group, a significant difference was observed in the LG, salivary gland (SG), eyeball, and kidney weight at P21 compared with P12 (Figure 1F). At $\mathrm{P} 21$, the changing ratio of the LG weight was significantly higher than that of the SG, eyeball, and kidney weight in the normal group. In the eyelid-sutured group, the changing ratio of the LG weight was significantly lower than in the age-matched normal controls. There was no significant difference in the changing ratio of the weight of the other organs and the whole body in the eyelid-sutured group compared with the age-matched normal group. The gross appearance of the LG from the eyelid-sutured pups at P21 was evidently smaller compared with the age-matched normal group (Figure 1F). The size of the SG and heart in the eyelid-sutured pups was the same as that of the agematched controls (P21).

These findings indicate that the occurrence of eye opening is important for the rapid growth of the LG during the postnatal period.

\section{Morphologic and Functional Changes of the Lacrimal Gland after Eye Opening}

The LG is a tubule-acinar exocrine gland that initiates by branching morphogenesis during the embryonic period and continues to develop during the postnatal period, during which it approaches maturity. ${ }^{29,30}$ The mature LG is composed of terminally differentiated acinar cells, myoepithelial cells, and ductal cells. ${ }^{29,31}$ These structures are organized as secretory lobes that cooperate to synthesize, modify, and secrete tear fluid. The morphologic changes in the acinar cells and myoepithelial cells of the LG, accompanying the occurrence of eye opening, were evaluated with two-photon, light, and electron microscopy.

Using two-photon microscopy, numerous secretory lobes composed of acinar cells and myoepithelial cells in the LG were observed in the normal group at P12 and P21 and the eyelid-sutured group at P21 (Figure 2A). In the normal mouse pups, individual secretory lobes were found enlarged at P21 compared with P12. The secretory lobes in the LG of the eyelid-sutured mouse pups at P21 were larger and smaller than those of the normal mouse pups at P12 and P21, respectively. Using two-photon microscopy at high magnification, enlarged acinar cells of the secretory lobes were observed in the LG of the normal group at P21 compared with P12 (Figure 2A). The myoepithelial cells surrounding the surface of the acinar cells grew with more elongated processes at P21 compared with P12 in the normal mouse pups. In the LG of the eyelid-sutured mouse pups at P21, a small size of acinar cells and a high density of myoepithelial cells on the LG surface were observed compared with the normal LG of P21. Most myoepithelial cell processes in the LG of eyelid-sutured pups at P21 were elongated compared with the normal LG at P12; however, some appeared shorter and thinner than the age-matched normal LG.

Using light microscopy to observe the hematoxylin and eosin-stained LG specimens, a gradual increase in the size of acinar cells with neonatal age was clearly observed in normal mouse pups, in particular in the cytoplasmic area of each individual acinar cell (Figure 2B). The acinar cell size 
was smaller in the LG of eyelid-sutured pups at P21 compared with the normal LG at P21, and the size was found to decrease between the normal P14 and P21 groups. Quantitative analysis of acinar cell size was performed at different postnatal ages in normal mouse and at P21 in eyelid-sutured mouse. Acinar cell size was found to significantly increase with postnatal age in the normal and eyelid-sutured groups compared with P12 (Figure 2B). In the eyelid-sutured mouse at P21, acinar cell size was approximately $75 \%$ of that in the age-matched normal mice, and significant differences were observed between the normal and eyelid-sutured mice at P21 (Figure 2B). Acinar cell density did not change throughout the postnatal age after eye opening in normal mice, and that of the eyelidsutured mice was the same as the age-matched normal mice (Figure 2B). These findings suggest that an enlargement of acinar cell size and not an increase in the number of acinar cells occurred in the LG during neonatal age after eye opening.

On transmission electron microscopy observation of the LG acinar cells of the normal mouse pups, a dilation of the stacked cisternae, composing the rough endoplasmic reticulum and the Golgi apparatus, as well as an increase in the number of secretory vesicles (SVs), was observed to be dependent on the age after eye opening (Figure 2C). In the LG of eyelid-sutured pups at P21, a narrow cisternae space of rough endoplasmic reticulum and Golgi apparatus and a small number of SVs were observed compared with agematched normal pups, and these findings resembled those of the normal LG at P12.

The gene expression levels of lactoperoxidase, an antibacterial protein synthesized on the rough endoplasmic reticulum and subsequently transported to the Golgi apparatus, ${ }^{32}$ were significantly higher in the LGs of the normal and eyelid-sutured mouse pups at P21 compared with those in the normal mouse pups at P12 (Figure 2D). Lactoperoxidase expression in the eyelid-sutured mouse pups was significantly lower than that in the age-matched normal mouse pups.

The changes in the tear components, which were synthesized in the LG and are secreted through SV-mediated exocytosis processes, ${ }^{33,34}$ were studied. Significant increases in the tear total protein concentration were observed in P21 compared with P14 in normal mice (Figure 2E). In the normal mouse pups, the tear lipocalin concentrations on P21 and P14 were similar. Compared with $\mathrm{P} 14$, a significant increase in tear lysozyme concentration was observed in the normal mice at P21. In terms of the concentration of total protein and lysozyme in the tear fluid, a significant difference was observed between the normal pups at P21 and the eyelid-sutured pups at P21, and no significant difference was observed between the normal pups at P14 and the eyelid-sutured pups at P21.

Together, these results suggest that morphologic maturation of the LG, accompanied by modification of tear secretory function, is accelerated after eye opening and delayed by eyelid suture.

\section{Corneal Sensory Experience Contributes to the Maturation of Lacrimal Gland and Neonatal Tearing}

The mature LG synthesizes and secretes tear fluid under exquisite neural regulation. ${ }^{35}$ The development of neural maturation is highly affected by early-life environmental exposure. ${ }^{12-14,36,37}$ Soon after postnatal eye opening, sensory experience as a consequence of environmental exposure plays a major role in the development of sensory organs and their underlying central neural connections. ${ }^{12-14,37,38}$ To obtain an adequate understanding of the potential influence of the sensory experience on the maturation of LG and neonatal tearing, the effect of stimulation or suppression of corneal sensory input on LG maturation was evaluated (Figure 3A). For the stimulation of the corneal sensory inputs in the mouse pups, eye drops of capsaicin $(1 \mu \mathrm{mol} / \mathrm{L})$ or menthol $(50 \mu \mathrm{mol} / \mathrm{L})$ were repeatedly administered to the mouse pups as each dosage has been reported to lead to sensory tearing by the activation of the neural loop via corneal sensory inputs. ${ }^{39}$ For the suppression of corneal sensory inputs, s.c. injections of a large amount of capsaicin $(50 \mathrm{mg} / \mathrm{kg}$ ) were administered at P5 and P6. This neonatal capsaicin treatment is a well-established animal model, and injection of a large amount of capsaicin during the neonatal period allows us to selectively destroy peripheral sensory nerve function as the capsaicin receptor is expressed in many sensory neurons at the early postnatal stage. ${ }^{40}$

The timing of the occurrence of eye opening did not differ significantly after sensory stimulation with capsaicin or menthol or sensory suppression in the normal group (data not shown). In the sensory stimulation group with capsaicin or menthol, the frequency of wipe behavior induced by the capsaicin or menthol eye drops was significantly increased compared with that by the saline eye drops (Figure 3B). In the sensory suppression group, the frequency of wipe behavior did not increase as a result of the capsaicin or menthol eye drops. Sensory stimulation with capsaicin and menthol did not change the corneal sensitivity compared with the age-matched vehicle group (Figure 3C). In the sensory suppression group, corneal sensitivity was significantly lower than in the normal group at P14 and P21 (Figure 3C and Supplemental Video S1).

At P21, sensory stimulation with capsaicin induced a significant increase in LG weight compared with the agematched normal mice (Figure 3D). The LG weight of sensory stimulation in the menthol group was the same as that of the vehicle group. In the sensory suppression group, the LG weight was significantly, approximately $30 \%$, lower than in the vehicle group. No significant differences in the weight of the other organs or the whole body were observed in the vehicle group, the sensory stimulation with capsaicin and menthol group, or the sensory suppression group. Figure $3 \mathrm{E}$ shows the gross appearance of the LG, SG, and 
A

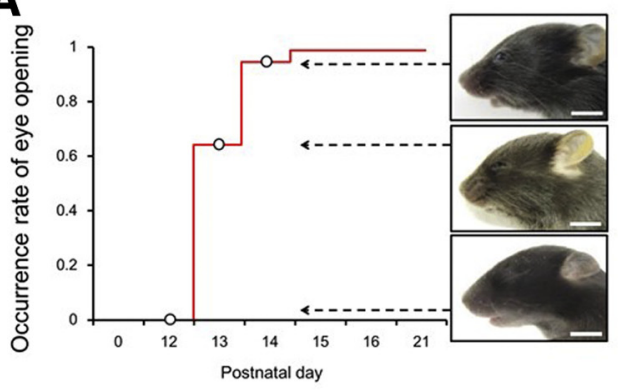

C

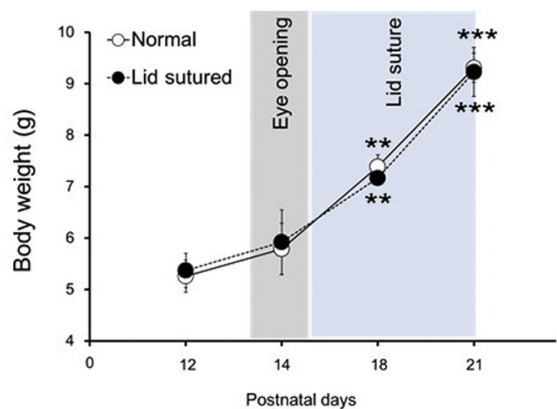

$\mathbf{E}$

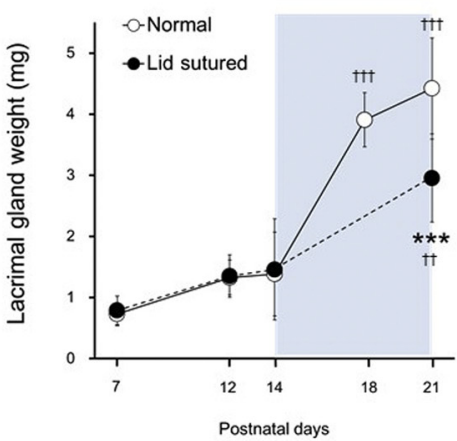

B

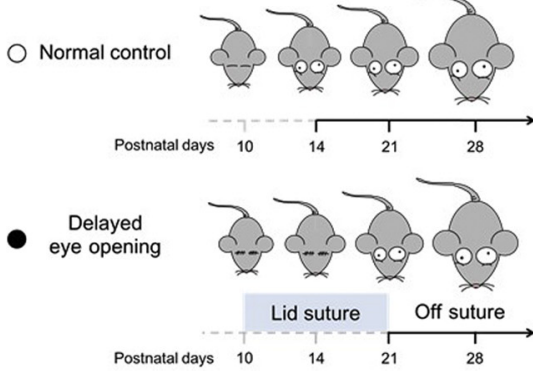

D
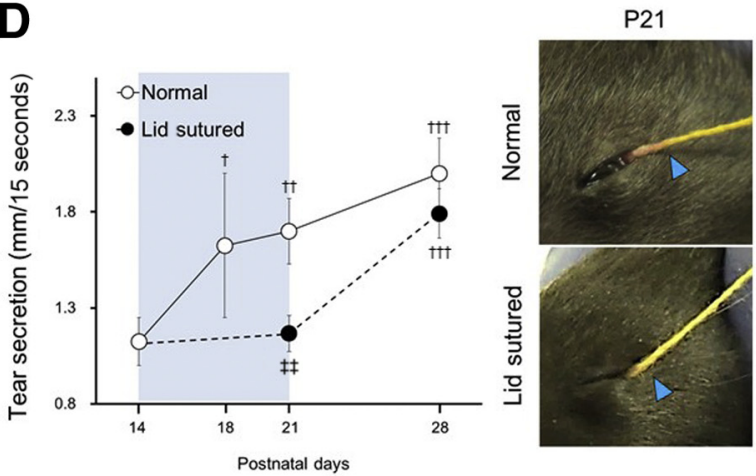

$\mathbf{F}$

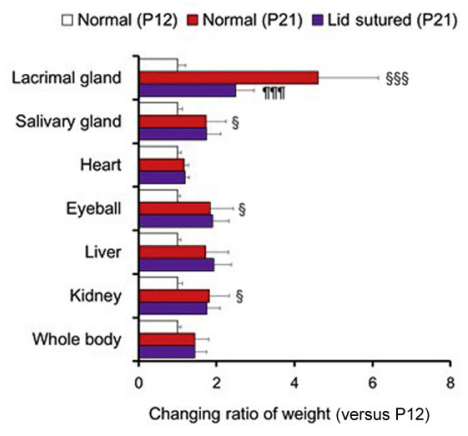

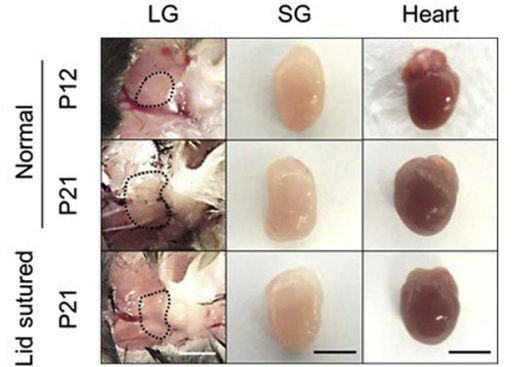

Figure 1 Eye opening accelerates the maturation of the lacrimal gland and neonatal tearing. A: Left panel: The occurrence rate of eye opening in normal wild-type mouse pups during the postnatal period. Right panel: Typical images of eyelid separation at postnatal days (P) 12, 13, and 14. B: Schematic of experimental schedule representing a comparison of the mouse pups, the eye-opening timing of which was delayed by 1 week by the binocular eyelid suture (eyelid-sutured group), and their healthy siblings without eyelid suture (normal control group). C and D: Changes in body weight (C) and tear secretion (D) in the normal control and eyelid-sutured groups. D: Left panel: Changes in tear secretion. Right panel: The representative images of tear secretion measured with a cotton thread at P21. The blue arrowheads show the wetted length by tear secretion. E: Changes in the lacrimal gland weight in the normal control and the eyelid-sutured groups. C-E: Gray and blue shading on the line chart indicate the duration of eye opening and eyelid suture, respectively. F: Changing ratio of tissue weight and body weight on P21. Left panel: Data show the ratio of tissue weight or body weight at P21 in the normal control and the eyelid-sutured group to that at P12 in the normal control. Right panel: The gross appearance of the lacrimal gland (LG), salivary gland (SG), and heart at P12 (normal control) and P21 (normal control and eyelid-sutured group). Dotted lines indicate the lacrimal gland. Data are expressed as means \pm SD (A, C, D, E, and F). $n=5$ to 10 mice (A, C, D, E, and F). ${ }^{*} P<0.05$, ${ }^{* *} P<0.01$, and ${ }^{* * *} P<0.001$ versus $\mathrm{P} 12 ;{ }^{\dagger} P<0.05,{ }^{\dagger \dagger} P<0.01$, and ${ }^{\dagger \dagger \dagger} P<0.001$ versus $\mathrm{P} 14$ (normal) or

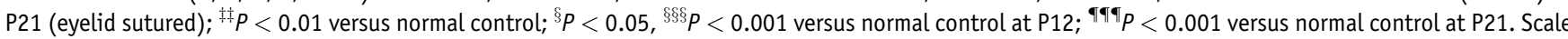
bars $=5 \mathrm{~mm}(\mathbf{A}$ and $\mathbf{F})$.

heart. Corresponding to the changes in tissue weight, the size of the LG was increased, unchanged, and reduced after sensory stimulation with capsaicin, sensory stimulation with menthol, and sensory suppression compared with the vehicle group, respectively. No obvious differences in the size of the SG or heart were observed among the vehicle group, the sensory stimulation with capsaicin group, the sensory stimulation with menthol group, or the sensory suppression group.

No significant differences in tear secretion were observed at P21 between the vehicle and sensory stimulation with capsaicin groups, although a significant difference was observed at P18 (Figure 3F). In the sensory stimulation with menthol group, tear secretion was consistent with that in the 
A

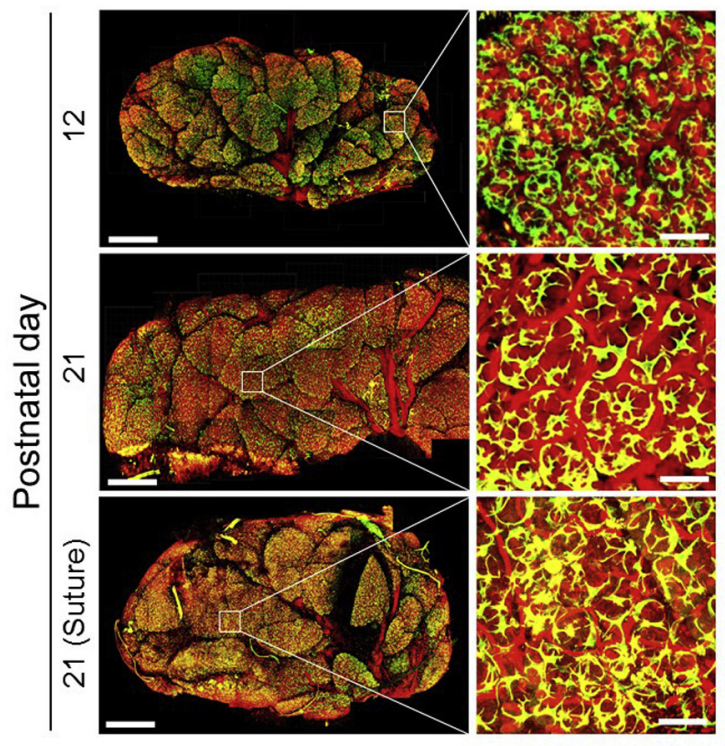

B

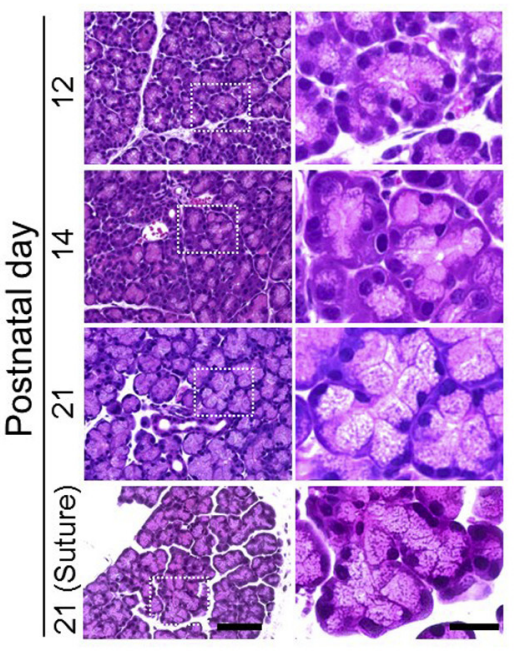

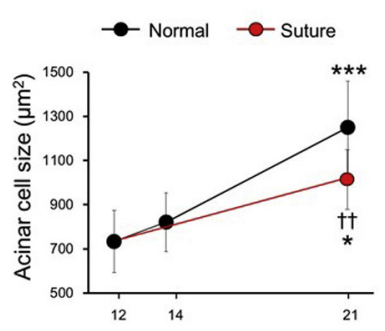

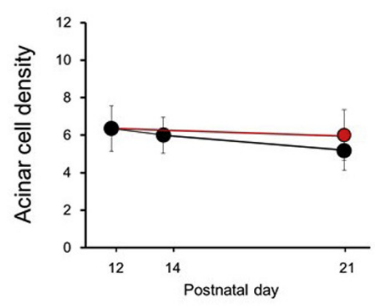

C
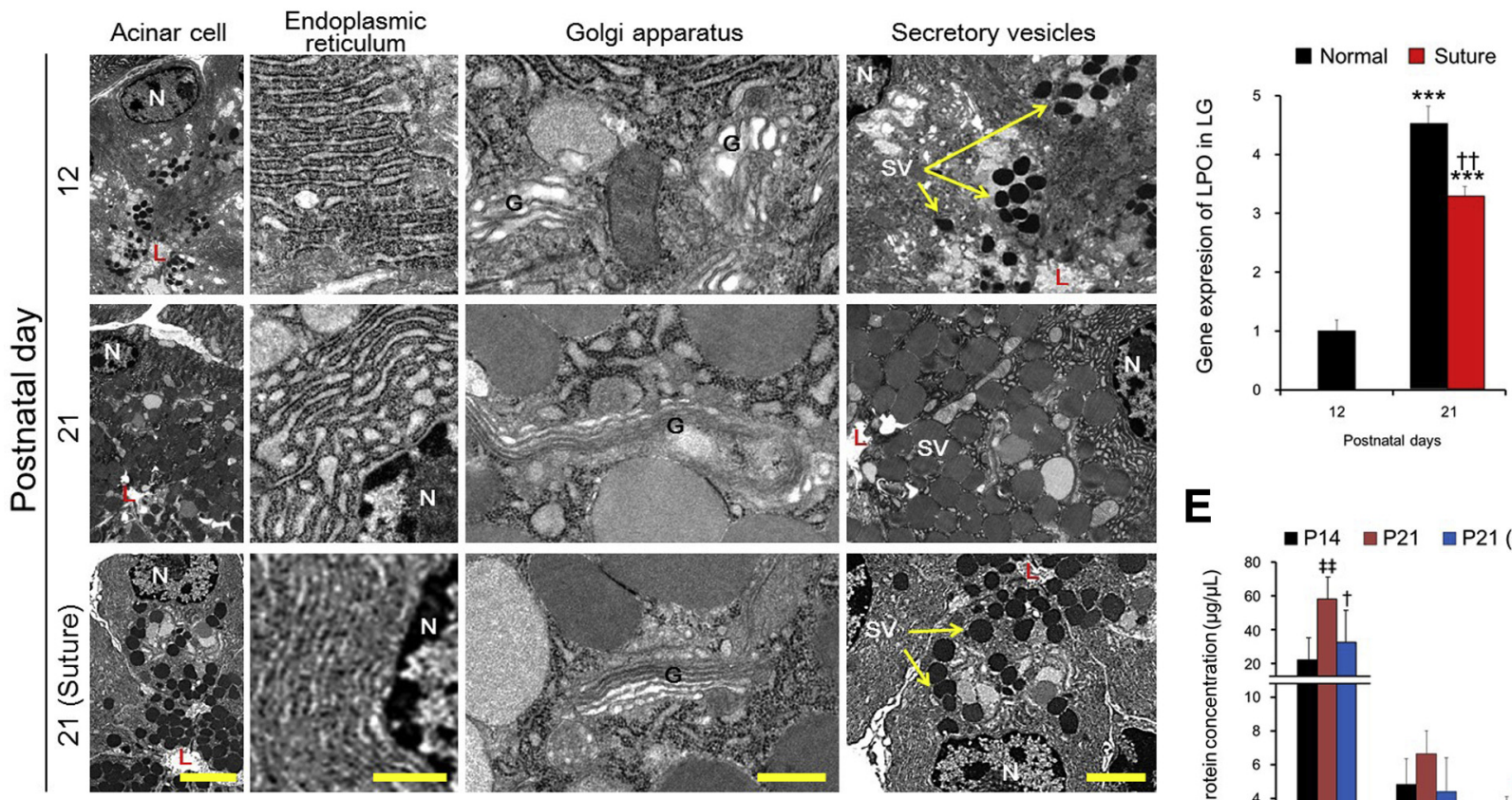

$\mathbf{E}$

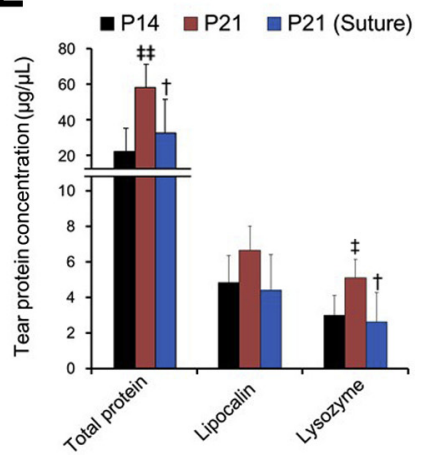

Figure 2 Morphologic and functional changes of neonatal lacrimal gland (LG) after eye opening. A: Two-photon microscopy of lacrimal gland isolated from normal mouse pups at postnatal days (P) 12 and 21 and from eyelid-sutured mouse pups at P21. Red and green fluorescence indicates the cellular surface of acinar cells and myoepithelial cells, respectively. B: Images show ematoxylin and eosin staining of the lacrimal gland of mouse pups. Charts indicate the quantitative analysis of changes in acinar cell size and acinar cell density, respectively. Acinar cell density was calculated as the occupied area ratio of the individual acinar cell/the whole lacrimal gland area $\left(\times 10^{-4}\right)$. C: Transmission electron microscopy observation of lacrimal gland acinar cells in normal mouse pups at P12 and P21 and eyelid-sutured mouse pups at P21. D: Gene expression of lactoperoxidase (LP0) in the lacrimal gland. E: Changes in tear components (total protein, lipocalin, and lysozyme). Data are expressed as means \pm SD (B, D, and $\mathbf{E}) . n=5$ to 10 mice or LGs $(\mathbf{B}, \mathbf{D}$, and $\mathbf{E}) .{ }^{*} P<0.05,{ }^{* * *} P<0.001$ versus $\mathrm{P} 12 ;{ }^{\dagger \dagger} P<0.01$ versus normal pups at $\mathrm{P} 21 ;{ }^{\ddagger} P<0.05,{ }^{\ddagger \ddagger} P<0.01$ versus $\mathrm{P} 14$. Scale bars: $200 \mu \mathrm{m}$ (A, left column); $50 \mu \mathrm{m}(\mathbf{A}$, right column, and $\mathbf{B}$, left column); $20 \mu \mathrm{m}$ (B, right column); $5 \mu \mathrm{m}$ (C, first column); $1 \mu \mathrm{m}$ (C, second column); $0.5 \mu \mathrm{m}$ (C, third column); $2 \mu \mathrm{m}$ (C, column). G, Golgi apparatus; $\mathrm{L}$, lumen; N, nucleus; SV, secretory vesicle. 


\section{A}

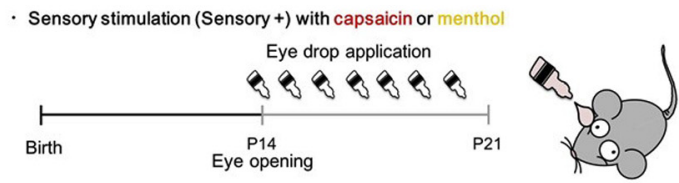

Sensory suppression (Sensory-)

Injection of vehicle or capsaicin (s.c., $50 \mathrm{mg} / \mathrm{kg}$ )

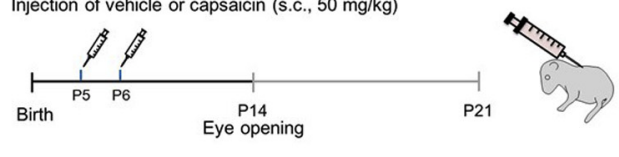

B

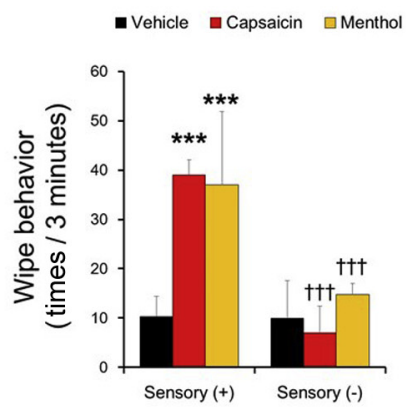

C

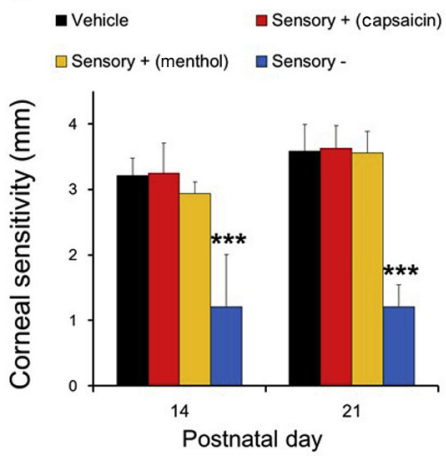

$\mathbf{F}$

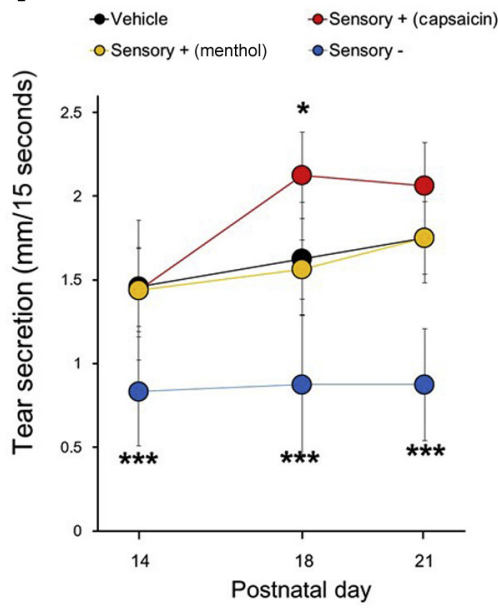

D

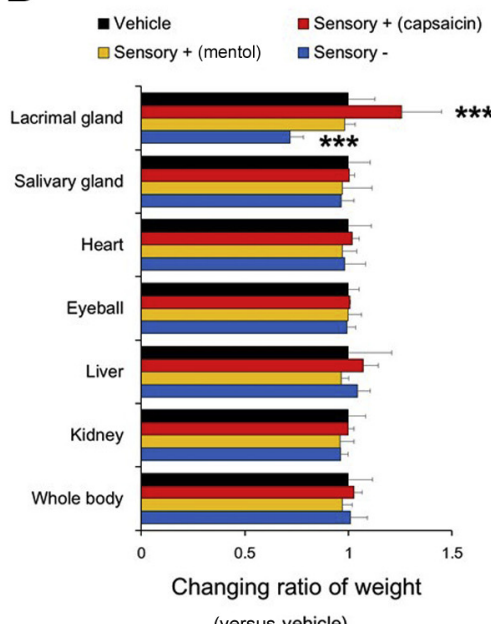

$\mathbf{E}$

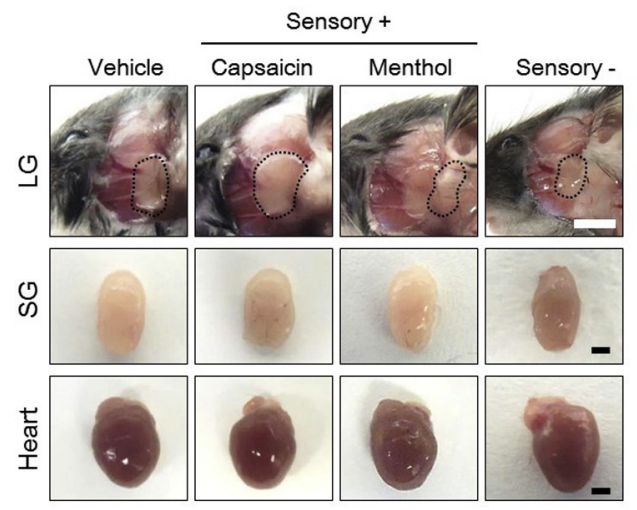

Figure 3 Involvement of corneal sensory stimulation with capsaicin in the maturation of lacrimal gland (LG) and neonatal tearing. A: Schematic of sensory stimulation and sensory suppression paradigm. Eye drops of capsaicin or menthol after eye opening and s.c. injection of capsaicin were performed for sensory stimulation and sensory suppression, respectively. B: Eye wipe behavior after eye drops of saline control, capsaicin, or menthol in sensory stimulation and sensory suppression groups. C: Corneal sensitivity of mouse pups in vehicle group, sensory stimulation with capsaicin group, sensory stimulation with menthol group, and sensory suppression group. D: Changing ratio of tissue weight and body weight on postnatal day (P) 21. Data show the ratio of tissue weight or body weight in sensory stimulation with capsaicin group, sensory stimulation with menthol group, and sensory suppression group compared with vehicle group. E: Gross appearance of LG, salivary gland (SG), and heart at P21. Dotted lines indicate the lacrimal gland. F: Changes in tear secretion after P14 in vehicle, sensory stimulation with capsaicin, sensory stimulation with menthol, and sensory suppression groups. Data are expressed as means \pm SD (B, C, D, and $\mathbf{F}) . n=10$ mice (B, C, D, and F). ${ }^{*} P<0.05,{ }^{* * *} P<0.001$ versus vehicle group; ${ }^{\dagger \dagger} P<0.001$ versus sensory $(-)$. Scale bars $=5 \mathrm{~mm}(\mathbf{E})$.

vehicle group throughout the experimental period. In the sensory suppression group, tear secretion was significantly lower than normal at P14, and remained significantly lower until P21.

These results suggest important roles of corneal sensory inputs via capsaicin-sensitive receptor in the maturation of LG and tear secretory activities.

\section{TRPV1 Channel Plays an Important Role in Neonatal Tearing}

To further understand the functions of corneal sensory experience in the maturation of neonatal tearing, the specific channels responding to corneal sensory signals were investigated. In adult animals, the involvement of corneal sensory signals via polymodal nociceptor and cold thermoreceptor in tearing has been reported. Cornel polymodal nociceptor is involved in pain signaling and triggers LG secretion. ${ }^{15,17,35,41}$ Corneal cold thermoreceptors are sensitive to slight alterations in corneal temperature at the normal corneal temperature and have been found to elicit a reflex stimulation to maintain the basal tearing rate. ${ }^{39}$ However, the molecular mechanisms whereby these neural inputs contribute to the maturation of neonatal tearing remain largely unsolved. Because capsaicin-activated nociceptors and menthol-activated cold thermoreceptors are characterized by the expression of nonselective transient receptor potential cation channels (TRPV1 and TRPM8) in sensory neurons, ${ }^{42-44}$ respectively, it was determined whether nociceptor TRPV1 and cold thermoreceptor TRPM8 contribute to the postnatal maturation of the LG using TRPVI and TRPM $8 \mathrm{KO}$ mouse pups. 
A

-WT -TRPV1 KO - TRPM8 KO

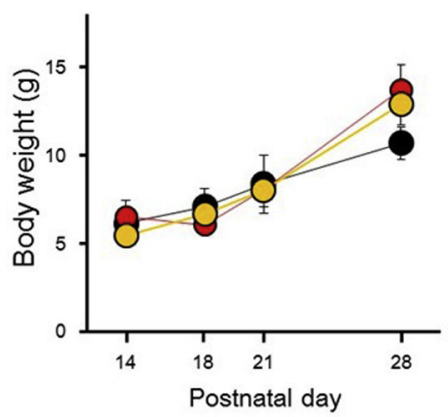

C

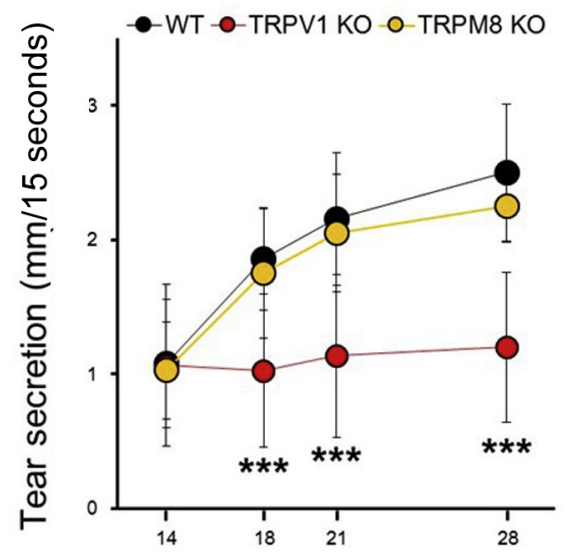

B

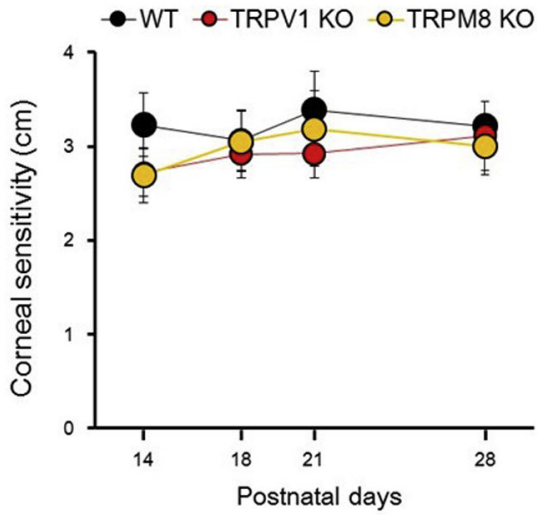

D
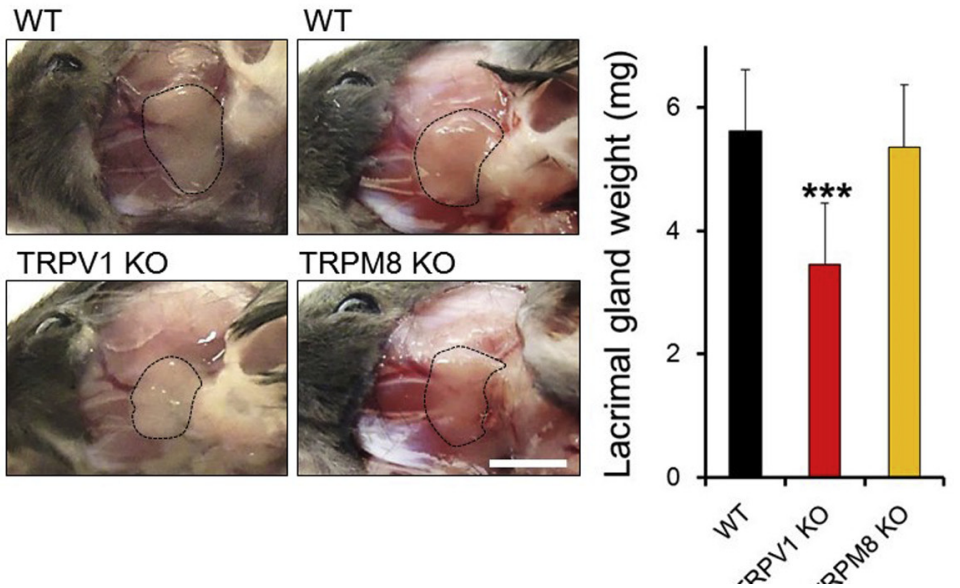

Figure 4 Transient receptor potential vanilloid 1 (TRPV1) knockout (K0) mice exhibit suppression of maturation of lacrimal gland and neonatal tearing A-C: Changes in body weight (A), corneal sensitivity (B), and tear secretion (C) in wild-type (WT) mouse pups, TRPV1 knockout mouse pups, and TRPM8 knockout mouse pups. D: Comparison of size and weight of the lacrimal gland among wild-type mouse pups, TRPV1 knockout mouse pups, and transient receptor potential melastatin 8 (TRPM8) knockout mouse pups. Images show the gross appearance of the lacrimal gland. Dotted line indicates the lacrimal gland. Bar chart shows the lacrimal gland weight at postnatal day 28. Data are expressed as means \pm SD (A-D). $n=6$ to 10 mice (A-D). ${ }^{* * *} P<0.001$ versus wild-type mouse. Scale bar $=5 \mathrm{~mm}$ (D).

The body weight gain of both the TRPVI KO mice and TRPM8 KO mice showed no difference compared with agematched WT mice during the experimental period (Figure 4A). In both the TRPVI KO and TRPM $8 \mathrm{KO}$ mice, corneal sensitivity was the same as in the age-matched WT mice from P14 to P28 (Figure 4B). At P14, no significant differences were observed in tear secretion among the WT, TRPV1 KO, or TRPM8 KO mice (Figure 4C). Tear secretion in the WT and TRPM8 KO mice was found to increase with postnatal age, and no significant difference in tear secretion was observed between the WT and TRPM $8 \mathrm{KO}$ mice from P14 to P28. However, the tear secretion of TRPVI KO mice was found to be unchanged from P14 to P28. Significant differences were observed at P18, P21, and P28 between the WT and TRPVI KO mice. The TRPVI KO mice exhibited a small size of the LG compared with the WT mice (Figure 4D). In the TRPM8 KO mice, the size of the LG was the same as that of the WT mice. The weight of the LG in the TRPVI KO mice was significantly lower than that in the
WT mice. No significant differences were observed in LG weight between the WT and TRPM $8 \mathrm{KO}$ mice.

Collectively, these findings demonstrate that the ocular surface sensory experience via TRPV1 plays a key role in the maturation of functional tear secretion during the postnatal period.

\section{Discussion}

Humans and mice undergo postnatal organ maturation at different paces. Nocturnal mammals with shorter gestation periods show a later postnatal opening of the eyelids, which allows for the healthy development of the immature ocular surface after birth. ${ }^{45}$ Furthermore, most murine organs or systems are relatively immature and in development at birth compared with humans. The starting times of aerobic respiration, fluid milk intake, and interactions with bacteria are different from those of environmental stimulation through the ocular surface; thus, the speed of postnatal 
organ maturation in mouse pups may be out of sync. The 2-week delay of eye opening after birth allows mouse pups to be a suitable model for investigating the influence of postnatal eye opening and the subsequent involvement of factors of extrauterine experiences on the maturation process of tearing. After birth, the growth of LG weight is initially modest; however, it becomes exponential after eye opening, whereas the growth of other vital organs sustains a steady rate. Binocular eyelid suture prolongs the eye closure time, thus posing a constraint to the sudden acceleration of the weight growth of the LG; however, it does not influence the weight growth of other vital organs.

In humans, the separation of eyelids occurs in utero; and newborns open their eyes immediately after birth, ${ }^{46,47}$ such that the tear volume of full-term newborns is highly increased within the first 4 postnatal months. ${ }^{10}$ The terminal organ maturation process of the fetus and the subsequent postnatal adaption are known to be the result of the timed and coordinated physiological regulation by internal and external determinants, including the levels of numerous hormones and changing intrauterine and extrauterine environments and experiences. The maturation process of vital organs is more adequately completed in humans at birth. Aerobic respiration, the acquisition of nutrients through food intake, interactions with bacteria, and the stimulation of the ocular surface occur simultaneously. Although small tear volumes and crying without tears are normal phenomena in full-term newborns at birth, lacrimal function matures quickly. Eye opening and the subsequent sensory stimulations subserve the maturation of neonatal tear secretory capacity.

The functional loop of the tearing unit is enhanced after eye opening. The lacrimal functional unit consists of the ocular surface, the LG, and the interconnecting innervation integrated within the brainstem. ${ }^{7}$ The exposure of the ocular surface to environmental stimuli after eye opening results in the switching on of nerve impulses through the innervations of the lacrimal functional unit. These results demonstrated that tear protein components, as well as the size and number of SVs in the LG, gradually increased after eye opening, indicating that the activation of tear protein synthesis is stimulated by the activation of sensory neurons after the switching on of the neural effect of eye opening. Before the separation of the eyelids, protein synthesis is limited because of the switching off of the sensory neural impulse, which is similar to sensory denervation. Denervation is often associated with the atrophic appearance and functional loss of the LG, SG, and muscles. Previous studies have shown that the loss of neural stimuli for saliva secretion during mastication induces a disuse atrophy of the parotid gland and a marked reduction in gland weight. ${ }^{48}$ Long-term physical inactivity, such as bed rest, leads to the deterioration of several body functions. ${ }^{49}$ The loss of sensory innervation may lead to an accumulation of SVs and a suppression of protein synthesis in the LG. ${ }^{50,51}$ Ferrari et $\mathrm{al}^{52}$ electrolyzed the ophthalmic branch of the trigeminal nerve and found a marked corneal opacity, reduced corneal innervation, and abolished corneal reflex, which resemble the experimental neurotrophic keratopathy associated with impaired tear function.

Lawrenson et $\mathrm{al}^{53}$ reported that the corneal sensation threshold of the ocular surface in neonates and infants was not significantly different to that in the adults. This correlates with our present data and demonstrates that the corneal reflex of mouse pups can be provoked immediately after eye opening. Moreover, the neonatal degeneration of capsaicin-sensitive sensory nerves in mouse pups delays the growth of the LG and is accompanied by a significant reduction in tear volume, indicating that the weakening of the functional neural loop could delay the growth in tear secretory activity and that capsaicin-sensitive sensory nerves play a role in the postnatal maturation of the lacrimal functional unit. Reasons for the retardation of neonatal tearing due to sensory suppression may be a decrease in the switching on of the neural loop, similar to disuse atrophy and sarcopenia. ${ }^{54}$ Although the s.c. injection of capsaicin may change the level of neurotrophic factors throughout the body, the effect of corneal stimulation by capsaicin instillation was investigated to determine the enhancement of corneal sensory afferents, and the sensory stimulation (turn up) of switching on the neural loop was found to promote the maturation of the lacrimal functional unit.

In this study, to determine which types of corneal sensory inputs play a role in the maturation of neonatal tearing, noxious stimuli via TRPV1 and physiological thermal stimuli via TRPM8 were studied. Using agonist and knockout mice for TRPVI and TRPM8, it was clearly demonstrated that the sensory experience via the TRPVIexpressing corneal sensory nerve plays a predominant role in the maturation of neonatal tearing.

Eye opening dramatically changes the ocular surface environment, including changes in tear film composition, exposure rate to external irritants, and thermal conditions. The main change in tear film composition due to eye opening is osmolality elevation, which is induced by an increase in the evaporation rate of the tear film from the ocular surface. ${ }^{55}$ Both the TRPV1 and TRPM8 channels are reported to be activated in response to osmolality elevation. ${ }^{56,57}$ Compared with TRPM8, which is mainly sensitive to cold temperatures, the sensitivity of TRPV1 to hypertonic stimuli is more toward physiologically relevant conditions. ${ }^{56,57}$ In addition, most of the sensory nerve fibers innervating the cornea are polymodal nociceptors; and TRPV1 plays an important role for sensory transduction, whereas only $9.5 \%$ of the sensory nerve fibers innervating the cornea are TRPM $8 .^{56}$ Thus, noxious stimulation, rather than osmotic and thermal stimulation, in response to environmental changes in the ocular surface during eye opening is a facilitative factor for the maturation of neonatal tearing. Further studies on other types of channels or sensory stimuli are required to identify other essential factors involved in neonatal tearing. 


\section{Acknowledgments}

We thank Dr. Mamoru Shibata (Keio University School of Medicine, Tokyo, Japan) for providing TRPM8 knockout mice and the Collaborative Research Resources (Keio University School of Medicine) for technical assistance.

K.J., T.I., and S.N. conceived and designed the study; K.J., T.I., Y.I., E.O., M.S., and H.S. performed the experiments and analyzed the data; M.I. performed the transmission electron microscopy analysis; K.K., M.I., H.T., and K.T. critically interpreted the results; K.J., T.I., S.N., and K.T. wrote the article; all authors participated in this study.

\section{Supplemental Data}

Supplemental material for this article can be found at http://doi.org/10.1016/j.ajpath.2019.05.015.

\section{References}

1. Fitzgerald M: The development of nociceptive circuits. Nat Rev Neurosci 2005, 6:507-520

2. Morton SU, Brodsky D: Fetal physiology and the transition to extrauterine life. Clin Perinatol 2016, 43:395-407

3. Xin M, Olson EN, Bassel-Duby R: Mending broken hearts: cardiac development as a basis for adult heart regeneration and repair. Nat Rev Mol Cell Biol 2013, 14:529-541

4. Newman JD: Neural circuits underlying crying and cry responding in mammals. Behav Brain Res 2007, 182:155-165

5. Sullivan DA, Stern ME, Tsubota K, Dartt DA, Sullivan RM, Bromberg BB (Eds): Lacrimal Gland, Tear Film, and Dry Eye Syndromes 3: Basic Science and Clinical Relevance. New York, NY: Springer US, 2002

6. Montes-Mico R, Cervino A, Ferrer-Blasco T, Garcia-Lazaro S, Madrid-Costa D: The tear film and the optical quality of the eye. Ocul Surf 2010, 8:185-192

7. Stern ME, Gao J, Siemasko KF, Beuerman RW, Pflugfelder SC: The role of the lacrimal functional unit in the pathophysiology of dry eye. Exp Eye Res 2004, 78:409-416

8. Botelho SY: Tears and the lacrimal gland. Sci Am 1964, 211:78-86

9. Sjogren H: The lacrimal secretion in newborn premature and fully developed children. Acta Ophthalmol 1955, 33:557-560

10. Esmaeelpour M, Watts PO, Boulton ME, Cai J, Murphy PJ: Tear film volume and protein analysis in full-term newborn infants. Cornea 2011, 30:400-404

11. Isenberg SJ, Apt L, McCarty J, Cooper LL, Lim L, Del Signore M: Development of tearing in preterm and term neonates. Arch Ophthalmol 1998, 116:773-776

12. Dorrn AL, Yuan K, Barker AJ, Schreiner CE, Froemke RC: Developmental sensory experience balances cortical excitation and inhibition. Nature 2010, 465:932-936

13. Katz LC, Shatz CJ: Synaptic activity and the construction of cortical circuits. Science 1996, 274:1133-1138

14. Hensch TK: Critical period plasticity in local cortical circuits. Nat Rev Neurosci 2005, 6:877-888

15. Acosta MC, Belmonte C, Gallar J: Sensory experiences in humans and single-unit activity in cats evoked by polymodal stimulation of the cornea. J Physiol 2001, 534:511-525

16. Acosta MC, Peral A, Luna C, Pintor J, Belmonte C, Gallar J: Tear secretion induced by selective stimulation of corneal and conjunctival sensory nerve fibers. Invest Ophthalmol Vis Sci 2004, 45:2333-2336
17. Belmonte C, Nichols JJ, Cox SM, Brock JA, Begley CG, Bereiter DA, Dartt DA, Galor A, Hamrah P, Ivanusic JJ, Jacobs DS, McNamara NA, Rosenblatt MI, Stapleton F, Wolffsohn JS: TFOS DEWS II pain and sensation report. Ocul Surf 2017, 15:404-437

18. Caterina MJ, Leffler A, Malmberg AB, Martin WJ, Trafton J, Petersen-Zeitz KR, Koltzenburg M, Basbaum AI, Julius D: Impaired nociception and pain sensation in mice lacking the capsaicin receptor. Science 2000, 288:306-313

19. Sakamoto R, Bennett ES, Henry VA, Paragina S, Narumi T, Izumi $Y$, Kamei Y, Nagatomi E, Miyanaga Y, Hamano H, Mitsunaga S: The phenol red thread tear test: a cross-cultural study. Invest Ophthalmol Vis Sci 1993, 34:3510-3514

20. Imada $T$, Nakamura $S$, Hisamura R, Izuta $Y$, Jin $K$, Ito $M$, Kitamura N, Tanaka KF, Mimura M, Shibuya I, Tsubota K: Serotonin hormonally regulates lacrimal gland secretory function via the serotonin type 3a receptor. Sci Rep 2017, 7:6965

21. Satoh Y, Oomori Y, Ishikawa K, Ono K: Configuration of myoepithelial cells in various exocrine glands of guinea pigs. Anat Embryol 1994, 189:227-236

22. Satoh Y, Sano K, Habara Y, Kanno T: Effects of carbachol and catecholamines on ultrastructure and intracellular calcium-ion dynamics of acinar and myoepithelial cells of lacrimal glands. Cell Tissue Res 1997, 289:473-485

23. Borges LF, Sidman RL: Axonal transport of lectins in the peripheral nervous system. J Neurosci 1982, 2:647-653

24. Aita M, Maeda T, Seo K: The effect of neonatal capsaicin treatment on the CGRP-immunoreaction in the trigeminal subnucleus caudalis of mice. Biomed Res 2008, 29:33-42

25. Kagawa $\mathrm{Y}$, Itoh $\mathrm{S}$, Shinohara $\mathrm{H}$ : Investigation of capsaicin-induced superficial punctate keratopathy model due to reduced tear secretion in rats. Curr Eye Res 2013, 38:729-735

26. Nakao A, Takahashi Y, Nagase M, Ikeda R, Kato F: Role of capsaicinsensitive C-fiber afferents in neuropathic pain-induced synaptic potentiation in the nociceptive amygdala. Mol Pain 2012, 8:51

27. Ko H, Cossell L, Baragli C, Antolik J, Clopath C, Hofer SB, MrsicFlogel TD: The emergence of functional microcircuits in visual cortex. Nature 2013, 496:96-100

28. Sullivan DA, Yee L, Conner AS, Hann LE, Olivier M, Allansmith MR: Influence of ocular surface antigen on the postnatal accumulation of immunoglobulin-containing cells in the rat lacrimal gland. Immunology 1990, 71:573-580

29. Farmer DT, Nathan S, Finley JK, Shengyang Yu K, Emmerson E, Byrnes LE, Sneddon JB, McManus MT, Tward AD, Knox SM: Defining epithelial cell dynamics and lineage relationships in the developing lacrimal gland. Development 2017, 144:2517-2528

30. Tsau C, Ito M, Gromova A, Hoffman MP, Meech R, Makarenkova HP: Barx2 and Fgf10 regulate ocular glands branching morphogenesis by controlling extracellular matrix remodeling. Development 2011, 138:3307-3317

31. Makarenkova HP, Dartt DA: Myoepithelial cells: their origin and function in lacrimal gland morphogenesis, homeostasis, and repair. Curr Mol Biol Rep 2015, 1:115-123

32. Anderson WA, Kang YH, Mohla S: Mammalian endogenous peroxidases as cellular markers and as biosynthetic endpoints of hormone-mediated activity: viewpoint from cytochemistry. Prog Histochem Cytochem 1979, 11:1-27

33. Farquhar MG: Progress in unraveling pathways of Golgi traffic. Annu Rev Cell Biol 1985, 1:447-488

34. Palade G: Intracellular aspects of the process of protein synthesis Science 1975, 189:347-358

35. Dartt DA: Neural regulation of lacrimal gland secretory processes: relevance in dry eye diseases. Prog Retin Eye Res 2009, 28:155-177

36. Bedrosian TA, Quayle C, Novaresi N, Gage FH: Early life experience drives structural variation of neural genomes in mice. Science 2018, 359:1395-1399

37. Verriotis M, Chang P, Fitzgerald M, Fabrizi L: The development of the nociceptive brain. Neuroscience 2016, 338:207-219 
38. Kobayakawa K, Kobayakawa R, Matsumoto H, Oka Y, Imai T, Ikawa M, Okabe M, Ikeda T, Itohara S, Kikusui T, Mori K, Sakano H: Innate versus learned odour processing in the mouse olfactory bulb. Nature 2007, 450:503-508

39. Parra A, Madrid R, Echevarria D, del Olmo S, Morenilla-Palao C, Acosta MC, Gallar J, Dhaka A, Viana F, Belmonte C: Ocular surface wetness is regulated by TRPM8-dependent cold thermoreceptors of the cornea. Nat Med 2010, 16:1396-1399

40. Cavanaugh DJ, Chesler AT, Braz JM, Shah NM, Julius D, Basbaum AI: Restriction of transient receptor potential vanilloid-1 to the peptidergic subset of primary afferent neurons follows its developmental downregulation in nonpeptidergic neurons. J Neurosci 2011, 31:10119-10127

41. Peirs C, Seal RP: Neural circuits for pain: recent advances and current views. Science 2016, 354:578-584

42. Caterina MJ, Schumacher MA, Tominaga M, Rosen TA, Levine JD, Julius D: The capsaicin receptor: a heat-activated ion channel in the pain pathway. Nature 1997, 389:816-824

43. Montell C, Birnbaumer L, Flockerzi V: The TRP channels, a remarkably functional family. Cell 2002, 108:595-598

44. Peier AM, Moqrich A, Hergarden AC, Reeve AJ, Andersson DA, Story GM, Earley TJ, Dragoni I, McIntyre P, Bevan S, Patapoutian A: A TRP channel that senses cold stimuli and menthol. Cell 2002, 108: 705-715

45. Meng Q, Mongan M, Carreira V, Kurita H, Liu CY, Kao WW, Xia Y: Eyelid closure in embryogenesis is required for ocular adnexa development. Invest Ophthalmol Vis Sci 2014, 55:7652-7661

46. Andersen H, Ehlers N, Matthiessen ME: Histochemistry and development of the human eyelids. Acta Ophthalmol 1965, 43:642-668

47. Knop E, Knop N, Millar T, Obata H, Sullivan DA: The international workshop on meibomian gland dysfunction: report of the subcommittee on anatomy, physiology, and pathophysiology of the meibomian gland. Invest Ophthalmol Vis Sci 2011, 52:1938-1978
48. Scott J, Gunn DL: Functional characteristics of atrophic parotid acinar cells from rats after liquid feeding. J Dent Res 1994, 73:1180-1186

49. Bortz WM 2nd: The disuse syndrome. West J Med 1984, 141: 691-694

50. Nguyen DH, Beuerman RW, Meneray M, Toshida H: Sensory denervation modulates eIF-2 alpha kinase expression in the rabbit lacrimal gland. Curr Eye Res 2006, 31:287-295

51. Nguyen DH, Beuerman RW, Toshida H: The effects of sensory and parasympathetic denervation on the kinases and initiation factors controlling protein synthesis in the lacrimal gland. Adv Exp Med Biol 2002, 506:65-70

52. Ferrari G, Chauhan SK, Ueno H, Nallasamy N, Gandolfi S, Borges L, Dana R: A novel mouse model for neurotrophic keratopathy: trigeminal nerve stereotactic electrolysis through the brain. Invest Ophthalmol Vis Sci 2011, 52:2532-2539

53. Lawrenson JG, Birhah R, Murphy PJ: Tear-film lipid layer morphology and corneal sensation in the development of blinking in neonates and infants. J Anat 2005, 206:265-270

54. Krishnan VS, White Z, McMahon CD, Hodgetts SI, Fitzgerald M, Shavlakadze T, Harvey AR, Grounds MD: A neurogenic perspective of sarcopenia: time course study of sciatic nerves from aging mice. J Neuropathol Exp Neurol 2016, 75:464-478

55. Liu H, Begley C, Chen M, Bradley A, Bonanno J, McNamara NA, Nelson JD, Simpson T: A link between tear instability and hyperosmolarity in dry eye. Invest Ophthalmol Vis Sci 2009, 50: 3671-3679

56. Quallo T, Vastani N, Horridge E, Gentry C, Parra A, Moss S, Viana F, Belmonte C, Andersson DA, Bevan S: TRPM8 is a neuronal osmosensor that regulates eye blinking in mice. Nat Commun 2015, 6:7150

57. Nishihara E, Hiyama TY, Noda M: Osmosensitivity of transient receptor potential vanilloid 1 is synergistically enhanced by distinct activating stimuli such as temperature and protons. PLoS One 2011, 6:e22246 